\title{
Elevated resting heart rate as independent in-hospital prognostic marker in COVID-19
}

\author{
Jennifer Vanoli ${ }^{1}$, Giacomo Marro ${ }^{2}$, Raffaella Dell'Oro ${ }^{1}$, Rita Facchetti ${ }^{1}$, \\ Fosca Quarti-Trevano ${ }^{1}$, Domenico Spaziani ${ }^{3}$, Guido Grassi ${ }^{1}$ \\ ${ }^{1}$ Clinica Medica, Department of Medicine and Surgery, University of Milano-Bicocca, Milan, Italy \\ ${ }^{2}$ Divisione di Medicina, Ospedale Bassini, Cinisello Balsamo, Milan, Italy \\ ${ }^{3}$ Unità Operativa Complessa di Cardiologia, Magenta Hospital, Magenta, Milan, Italy
}

\begin{abstract}
Background: Scarce and non-homogeneous data are available on the prognostic value of clinic heart rate (HR) in coronavirus disease 2019 (COVID-19).

Methods: The present study evaluated in 389 patients hospitalized for COVID-19 the in-hospital prognostic value of resting $H R$, assessed over different time periods, i.e., at hospital admission, during initial 3 days and 7 days of hospitalization.

Results: Results show that assessment of this hemodynamic variable during hospitalization provides information on the clinical outcome of the patients, greater HR values being associated with a worse inhospital prognosis. The prognostic value of elevated HR during COVID-19: 1) was independent on other confounders such as age, gender, comorbidities and fever, 2) appeared to be strengthened by repeated measurements of HR during the initial 3/7 days of hospitalization, and 3) was detectable in patients in which the therapeutic intervention did not include drugs, such as beta-blockers, calcium antagonists, digoxin, ivabradine and antiarrhythmic compounds known to interfere with HR.

Conclusions: Heart rate may represent an important marker of a patient's outcome in COVID-19. (Cardiol J 2022; 29, 2: 181-187)

Key words: coronavirus disease 2019 (COVID-19), heart rate, sympathetic nervous system, prognosis
\end{abstract}

\section{Introduction}

Several studies have shown that age, gender, obesity and comorbidities represent the main predictors of coronavirus disease 2019 (COVID-19) infection severity [1-4] and that patients with high mortality and morbidity rate also developed a hyperinflammatory syndrome [5]. Severe acute respiratory syndrome coronavirus 2 (SARS-CoV-2) infection has been associated with multiple abnormalities of the autonomic control of the circulation, a phenomenon known as "long COVID" [6]. Activation of sympathetic cardiovascular drive together with attenuation of vagal cardiac influences caused by the virus itself and by inflammatory cytokines reaction which can lead to a serious autonomic imbalance contributing to worsen the clinical course of the SARS-CoV-2 infection [6-9]. The main clinical signs of these autonomic alterations are represented by the occurrence of elevated resting heart rate (HR) values and frequently by the development of a resting tachycardia. Both these alterations, which have been reported in cardiovascular and non-cardiovascular disease as reliable markers of an increased adrenergic neural drive to the heart $[10,11]$, have been suggested to be associated with a poor prognosis in SARS-CoV-2 infection. However, the few studies published so

Address for correspondence: Prof. Guido Grassi, Clinica Medica, University Milano-Bicocca, Via Pergolesi 33, 20052 Monza, Italy, tel: +39039 2333357, e-mail: guido.grassi@unimib.it

Received: 21.09.2021 Accepted: 2.12.2021 Early publication date: 18.02.2021

This article is available in open access under Creative Common Attribution-Non-Commercial-No Derivatives 4.0 International (CC BY-NC-ND 4.0) license, allowing to download articles and share them with others as long as they credit the authors and the publisher, but without permission to change them in any way or use them commercially. 
far examining the relationships between tachycardia and prognosis after SARS-CoV-2 disease provided results frequently non-homogeneous and in the majority of cases were based only on a single evaluation of HR at hospital admission [12-14]. The aim of the present study was to investigate in a systematic and standardized way whether, and to what extent HR assessed over different time periods during hospitalization (hospital admission and during the first 3 and 7 days of hospitalization) represents a prognostic marker of severity of SARS-CoV-2 infection in terms of care intensity during hospitalization. A secondary aim was to determine the impact of HR assessed over the above-mentioned different time periods on Kaplan-Meyer survival curves.

\section{Methods}

\section{Study population}

This retrospective analysis was performed to investigate the clinical characteristics of $389 \mathrm{pa}-$ tients with SARS-CoV-2 infection admitted to the medical hospital units of the institutions involved in the study between March $13^{\text {th }}$ and December $6^{\text {th }}, 2020$. A diagnosis of SARS-CoV-2 infection was confirmed by a positive reverse transcriptase polymerase chain reaction of a specimen collected via a nasopharyngeal swab. Demographic, anamnestic, anthropometric, clinical, laboratory, instrumental, treatment and outcome data were extracted from electronic medical records. Anamnestic data referred to history of cigarette smoking and comorbidities (e.g., chronic obstructive pulmonary disease, obstructive sleep apnea, hypertension, diabetes mellitus, chronic heart failure, coronary artery disease, history of atrial fibrillation or flutter, history of implantable cardioverter-defibrillator or permanent pacemaker implant, chronic impaired renal function and liver disease). Treatment data also included information on the assumption of drugs potentially active on HR (beta-blockers, calcium channel blockers, antiarrhythmic compounds, ivabradine and digoxin) both before and during hospitalization and on SARS-CoV-2 specific treatment prescribed according to clinical needs. Detection of an overweight/obese state at hospital admission was also reported. For each patient we noted the concomitant number of comorbidities $\geq 2$ or $<2$.

Assessment of resting HR was performed at the time of the hospital admission and repeated each morning during the first $3 / 7$ days of hospitalization. It was obtained through physical examination (palpatory method of the radial artery, average of 3 measurements performed within $10 \mathrm{~min}$ ) and reported in clinical diaries. For each HR measurement we concomitantly evaluated whether the patient was febrile (body temperature $>37.5^{\circ} \mathrm{C}$ ). Clinical data also included chest X-ray and clinic blood pressure values recorded at the time of hospital admission. We categorized each participant according to the highest care intensity needed during hospitalization, defining four severity outcome classes of SARS-CoV-2 infection as follows: 1) severity outcome class I, no need for oxygen support/need for low flow oxygen therapy (maximum $35 \% \mathrm{FiO}_{2}$ delivered through nasal cannula or Venturi mask), 2) severity outcome class II, need for high flow oxygen therapy/continuous positive airway pressure, 3) severity outcome class III, transfer to the intensive care unit, and 4) severity outcome class IV, and death.

\section{Statistical analysis}

Heart rate values were evaluated both as the first measurement at the hospital admission and as the means of HR values over 3-days and 7-days from hospitalization respectively, before inclusion of the patient in one of the severity classes described previously. Patient characteristics were analyzed by descriptive statistics. For continuous variables, the mean and standard deviation (mean $\pm \mathrm{SD}$ ) or the median and interquartile range were calculated. For discrete (categorical) variables, percentages (\%) in each category were calculated. The statistical significance of the between-group differences was assessed by ANOVA, the Kruskal-Wallis test, $\chi^{2}$ test or the Fisher exact test. Trends between the severity outcome classes and clinical variables were tested using integer values $(1,2$, 3 and 4) in a linear regression model or CochranArmitage test for trend. Trends were also adjusted for sex, age, number of comorbidities greater than 2 and number of days in which body temperature $>37.5^{\circ} \mathrm{C}$. The Kaplan-Meier survival curves based on all cause death or intensive care unit admission were calculated for the groups of patients displaying at various periods of hospitalization (hospital admission, first 3-days and 7-days of hospitalization) HR values below and above 80 beats/min, i.e., the value indicated by the European Society of Cardiology/European Society of Hypertension guidelines as the cutoff above which cardiovascular risk is clearly elevated [15]. All p values were two-sided and a p $<0.05$ was considered as statistically significant. The statistical analysis was carried out by the SAS package (SAS Institute, Cary, NC, USA). 


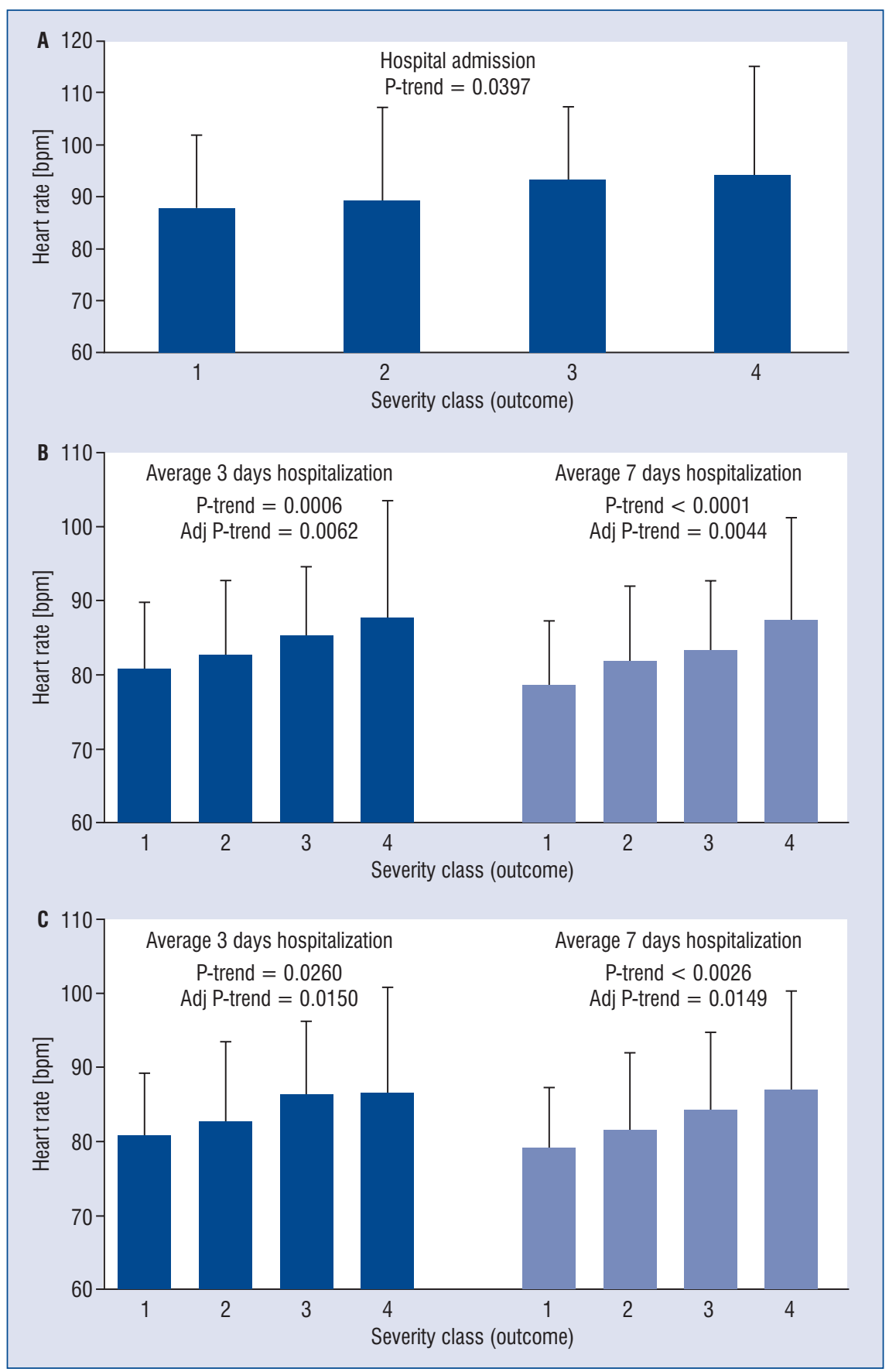

Figure 1. Relationships between the outcome of SARS-CoV-2 infection and the first assessment of heart rate at hospital admission ( $n=389, A$ ), mean heart rate values referred to the first 3-days and 7-days of hospitalization $(n=217, B)$ and mean heart rate values referred to the first 3-days and 7-days of hospitalization in patients not receiving drugs potentially interfering with heart rate $(n=118, C)$. Data are shown as means \pm standard deviation.

\section{Results}

The present analysis was divided into three steps (Fig. 1). The association between resting HR and clinical outcome of SARS-CoV-2 infection assessing HR values was initially examined at hos- pital admission. HR assessment was then repeated taking into account values recorded during the first 3 -days and 7-days of hospitalization, respectively. Finally, the evaluation was repeated excluding patients treated with drugs which potentially affected HR. In the study population as a whole $(n=389)$, 
Table 1. Baseline characteristics of patients with SARS-CoV-2 infection at the time of the hospital admission.

\begin{tabular}{|c|c|c|c|c|c|c|}
\hline \multirow[t]{2}{*}{ Variable } & \multicolumn{5}{|c|}{ Outcome } & \multirow[t]{2}{*}{$P$ trend } \\
\hline & Overall & $\begin{array}{l}\text { Severity } \\
\text { class } 1\end{array}$ & $\begin{array}{l}\text { Severity } \\
\text { class } 2\end{array}$ & $\begin{array}{c}\text { Severity } \\
\text { class } 3\end{array}$ & $\begin{array}{l}\text { Severity } \\
\text { class } 4\end{array}$ & \\
\hline Number of patients & 389 & 121 & 142 & 43 & 83 & \\
\hline \multicolumn{7}{|l|}{ Demographic characteristics } \\
\hline Age [years] & $67.4 \pm 14.6$ & $62.4 \pm 16.1$ & $67.8 \pm 13.5$ & $61.3 \pm 9.4$ & $77 \pm 11.2$ & $<0.0001$ \\
\hline Male & $68.4 \%$ & $62.8 \%$ & $70.4 \%$ & $83.7 \%$ & $65.1 \%$ & 0.4405 \\
\hline Current smoking & $4.5 \%$ & $5.9 \%$ & $2.2 \%$ & $2.3 \%$ & $7.7 \%$ & 0.5897 \\
\hline \multicolumn{7}{|l|}{ Comorbirdities } \\
\hline $\mathrm{BMI} \geq 25 \mathrm{~kg} / \mathrm{m}^{2}$ & $39.1 \%$ & $33.6 \%$ & $44.9 \%$ & $42.5 \%$ & $35 \%$ & 0.9355 \\
\hline Hypertension & $52.4 \%$ & $43 \%$ & $54.9 \%$ & $51.2 \%$ & $62.7 \%$ & 0.0106 \\
\hline Diabetes mellitus & $22.2 \%$ & $16.7 \%$ & $22.5 \%$ & $20.9 \%$ & $30.1 \%$ & 0.0332 \\
\hline Chronic kidney disease & $10.3 \%$ & $10.7 \%$ & $7.7 \%$ & $0 \%$ & $19.3 \%$ & 0.1332 \\
\hline COPD & $5.7 \%$ & $4.1 \%$ & $5.6 \%$ & $4.7 \%$ & $8.4 \%$ & 0.2321 \\
\hline Heart failure & $8.5 \%$ & $7.4 \%$ & $6.4 \%$ & $0 \%$ & $18.1 \%$ & 0.0264 \\
\hline Coronary heart disease & $14.1 \%$ & $15.7 \%$ & $9.2 \%$ & $2.3 \%$ & $26.5 \%$ & 0.0745 \\
\hline Obstructive sleep apnea & $3.1 \%$ & $1.7 \%$ & $2.8 \%$ & $7 \%$ & $3.6 \%$ & 0.2601 \\
\hline ICD/PPM & $3.1 \%$ & $1.7 \%$ & $4.2 \%$ & $0 \%$ & $4.8 \%$ & 0.3839 \\
\hline Atrial fibrillation or flutter history & 14.7 & 10.7 & 12.7 & 0 & 31.3 & 0.0007 \\
\hline Liver disease & $1.3 \%$ & $1.7 \%$ & $1.4 \%$ & $0 \%$ & $1.2 \%$ & 0.3227 \\
\hline Number of comobidities $\geq 2$ & $50.1 \%$ & $39.7 \%$ & $52.1 \%$ & $34.9 \%$ & $69.9 \%$ & 0.0004 \\
\hline \multicolumn{7}{|l|}{ Admission profile } \\
\hline Body temperature $>37.5^{\circ} \mathrm{C}$ & $28.7 \%$ & $20.7 \%$ & $29.3 \%$ & $42.9 \%$ & $32.5 \%$ & 0.0357 \\
\hline $\mathrm{PaO}_{2} / \mathrm{FiO}_{2}$ & $\begin{array}{c}276 \\
(200-323)\end{array}$ & $\begin{array}{c}328 \\
(300-368.5)\end{array}$ & $\begin{array}{c}261 \\
(222-304)\end{array}$ & $\begin{array}{c}210 \\
(121-266)\end{array}$ & $\begin{array}{c}190 \\
(134-266)\end{array}$ & $<0.0001$ \\
\hline Systolic BP [mmHg] & $138 \pm 21.8$ & $136.3 \pm 21.4$ & $138.8 \pm 23$ & $132.9 \pm 18$ & $142.2 \pm 21.7$ & 0.1700 \\
\hline Diastolic BP [mmHg] & $79.7 \pm 13.7$ & $79.7 \pm 13$ & $80 \pm 14.8$ & $76.9 \pm 10.7$ & $80.6 \pm 14.4$ & 0.9538 \\
\hline Heart rate $[\mathrm{bpm}]$ & $90.4 \pm 17.1$ & $88 \pm 13.9$ & $89.3 \pm 17.9$ & $93.4 \pm 13.8$ & $94.3 \pm 20.7$ & 0.0049 \\
\hline WBC count [cell $/ \mu \mathrm{L}]$ & $\begin{array}{c}6960 \\
(5030-10240)\end{array}$ & $\begin{array}{c}6460 \\
(4710-8230)\end{array}$ & $\begin{array}{c}6925 \\
(5270-10475)\end{array}$ & $\begin{array}{c}7300 \\
(5160-9490)\end{array}$ & $\begin{array}{c}8140 \\
(5440-12260)\end{array}$ & $<.0001$ \\
\hline Hs-CRP concentration [mg/dL] & $\begin{array}{c}6.5 \\
(2.1-13)\end{array}$ & $\begin{array}{c}2.3 \\
(0.8-6.8)\end{array}$ & $\begin{array}{c}6.9 \\
(3.6-13)\end{array}$ & $\begin{array}{c}10.6 \\
(5.9-13.7)\end{array}$ & $\begin{array}{c}12.1 \\
(5.2-16.5)\end{array}$ & 0.8125 \\
\hline Creatinine [mg/dL] & $1.4 \pm 1.3$ & $1.3 \pm 1.6$ & $1.3 \pm 1.2$ & $1.2 \pm 0.6$ & $1.7 \pm 1.3$ & 0.0476 \\
\hline eGFR $\left[\mathrm{mL} / \mathrm{min} / 1.73 \mathrm{~m}^{2}\right]$ & $58.6 \pm 27.1$ & $66.0 \pm 27.6$ & $58.6 \pm 24.3$ & $57.1 \pm 20.2$ & $48.8 \pm 30.7$ & $<0.0001$ \\
\hline
\end{tabular}

Data are shown as means \pm standard deviation or median (interquartile range) or as percent values; BMI — body mass index; BP — blood pressure; COPD - chronic obstructive pulmonary disease; hs-CRP - high-sensivity C-reactive protein; e-GFR - estimated glomerular filtration rate (MDRD formula); IDC/PPM — implantable cardioverter-defibrillator/permanent pacemaker placement; WBC — white blood cell

$121(31 \%)$ participants were categorized in severity outcome class I, while the remaining 268 were in the other classes > I, i.e., $142(37 \%)$ class II, $43(11 \%)$ class III and $83(21 \%)$ class IV (Table 1). Typical chest-X ray findings included pulmonary consolidation or ground-glass opacities with different pulmonary distribution. Mean age amounted to $67.4 \pm 14$ years, $264(68 \%)$ were males and $125(32 \%)$ females. $50 \%$ of the patients displayed at least two comorbidities and $42 \%$ were under pharmacological treatment with drugs acting on HR. The deceased patients aged $77.1 \pm 11$ years, they displayed a high number of comorbidities and elevated serum levels of inflammatory markers.

Assessment of resting HR at hospital admission was available in 381 patients, which displayed an average value amounting to $90.1 \pm 17$ beats/ $/$ min. For each class increase in maximum care in- 
tensity, a corresponding significant increase in HR was observed ( $\mathrm{p}$ value trend $=0.0397$ ) $($ Fig. 1A). HR measurements during hospitalization were available in 217 patients, $24 \%$ were categorized in severity outcome class I, $27 \%$ in class II, $14 \%$ in class III and 35\% class IV. Baseline characteristics were representative of the overall population. For each class increase in maximum care intensity, a corresponding significant increase in HR was observed which considered data collected during first 3 days of hospitalization ( $p$ value trend $<0.0006)$ and more so during the first 7 days of hospitalization ( $\mathrm{p}$ value trend $<0.0001$ ) (Fig. 1B). The significant trend was maintained after adjustment for age, gender, number of comorbidities and number of days during which body temperature was $>37.5^{\circ} \mathrm{C}$.

The same analysis was performed in the 118 patients not receiving drugs potentially acting on HR both before and during hospitalization. Even in this case, a significant trend was observed between the increase in the average HR and the severity of the infection taking into account data collected during the first 3 days ( $\mathrm{p}$ value trend $=0.02$ ) 7 days ( $\mathrm{p}$ value trend $=0.002$ ) of hospitalization (Fig. 1C).

Finally, as shown in Figure 2, the Kaplan-Meier curves for survival based on HR assessment at hospital admission or during the 3 -days and 7 -days of hospitalization displayed during the follow-up survival probability curves were quite similar, with a decreased survival rate in the patients displaying greater clinic HR values.

\section{Discussion}

The aim of the present study was to determine in patients hospitalized for SARS-CoV-2 disease the prognostic relevance of resting HR evaluated at different time periods during hospitalization. The results show that assessment of this hemodynamic variable during hospitalization provides information on the clinical outcome of the patients, greater HR values being associated with a worse in-hospital prognosis. Additional results of the study are represented by the finding that the prognostic value of elevated HR during COVID-19: 1 ) is independent on other confounders such as age, gender, comorbidities and fever, 2) appears to be strengthened by repeated measurements of $\mathrm{HR}$ during the initial 3/7 days of hospitalization, and 3) can be detected also in the groups of patients in which pharmacological treatment was not based on drugs, such as beta-blockers, calcium antagonists, digoxin, ivabradine and antiarrhythmic compounds known to interfere with HR. The current analysis on the relevance of HR as in-hospital prognostic marker provides an additional result. That is that the assessment of HR based on 7-days evaluation may allow obtaining an estimation of survival probability more sensitive and accurate than the other evaluations based on HR at admission and during the first 3 days of hospitalization.

It is well known that the increase in HR and the development of a resting tachycardia represents the result of an increased sympathetic cardiac drive with a concomitant reduction of the parasympathetic influences to the heart [11]. This is supported by the evidence that in a number of cardiovascular and non-cardiovascular diseases there is a close significant direct relationship between different markers of sympathetic cardiovascular drive (venous plasma norepinephrine, cardiac norepinephrine spill over, direct recording of efferent postganglionic sympathetic nerve traffic to the skeletal muscle) and resting HR [10, 16, 17]. The autonomic alterations described above (which may have a reflex origin mediated by arterial baroreceptor and chemoreceptor alterations $[16,17])$ may occur in COVID-19 and thus may be responsible for the HR elevation detected in diseased patients.

\section{Conclusions}

The present study has strengths and limitations. As mentioned above the strengths are: 1 ) the value of $\mathrm{HR}$ as prognostic marker in COVID-19 patients even after data adjustment for confounders, 2 ) the data analysis performed taking into account the number of comorbidities, and 3) the separate analysis of the data done in patients untreated with drugs known to interfere with HR. The limitations include: 1 ) the relatively small number of patients included in the study, a limitation which may also depend on the strict criteria adopted in patient selection, 2) the fact that the analysis was limited to the first 7 days of hospitalization only, no further evaluation was made after this time period, 3 ) the consideration that a comparison of the Kaplan-Meyer curves based on HR evaluations at different time periods is limited to the reduced number of patients available particularly for the 3 and 7 day assessment, and 4) the fact that in the present study, the mechanisms through which elevated HR may adversely affect prognosis in COVID-19 patients were not investigated. However, in other diseases, such as in heart failure, coronary artery disease and hypertension conclusive evidence has been provided that elevated HR values: 


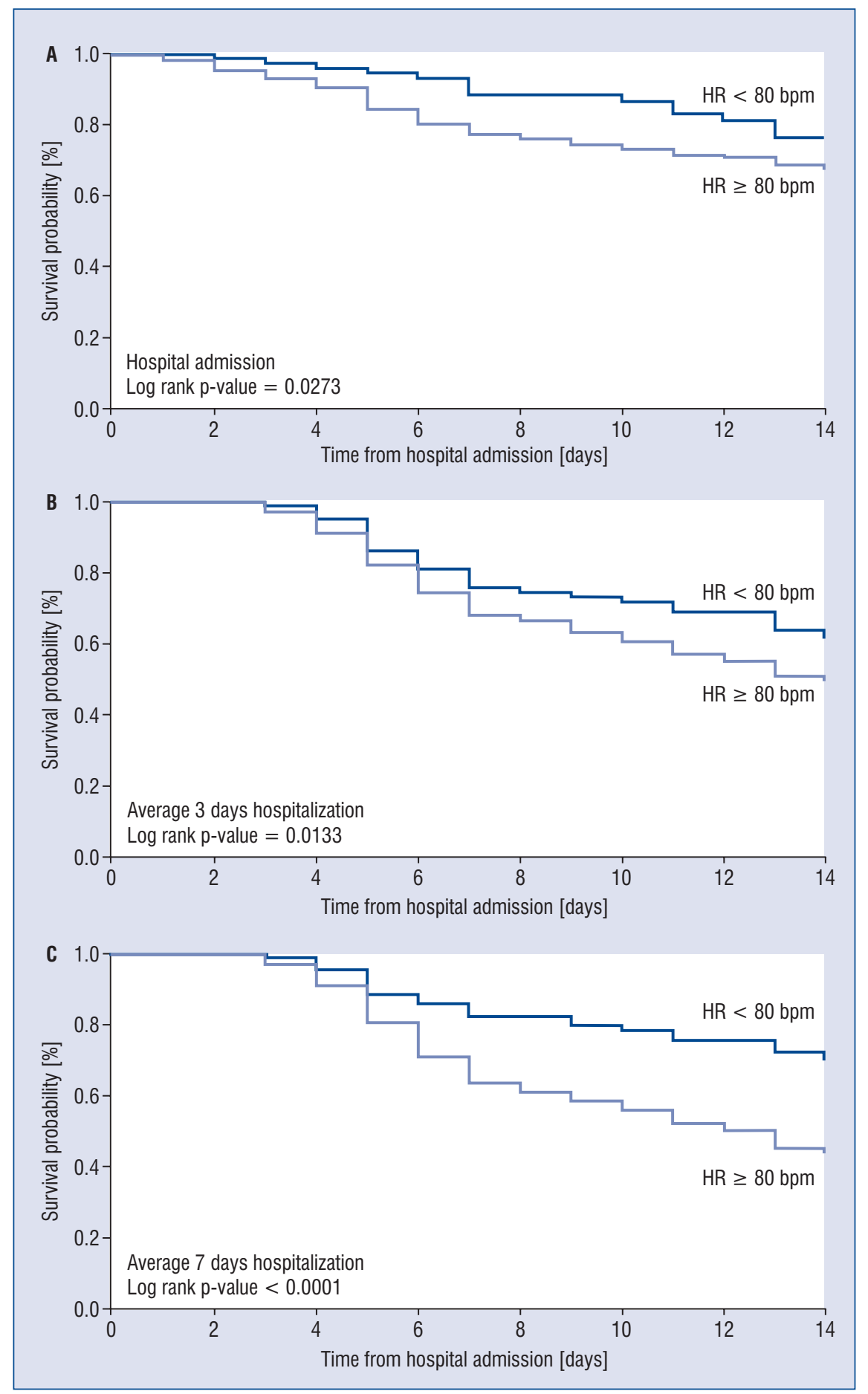

Figure 2. Kaplan-Meyer curves for survival without poor outcome (death or intensive care unit admission) in patients with resting heart rate (HR) below or above 80 beats/minute [bpm] based on measurements of HR performed at hospital admission (A), during the first 3 days (B) or 7 days $(\mathbf{C})$ of hospitalization.

1) increase cardiac work and myocardial oxygen demand [17], 2) augment arterial wall stress [16, 17], 3) decrease arterial distensibility, and 4) favour the development and progression of the coronary atherosclerotic plaque $[11,16]$. All these pathophysi- ological consequences may be responsible also in COVID-19 for the adverse prognostic impact of elevated HR values reported in the present study.

Conflict of interest: None declared 


\section{References}

1. Iaccarino G, Grassi G, Borghi C, et al. Age and multimorbidity predict death among COVID-19 patients. Hypertension. 2020; 76(2): 366-372, doi: 10.1161/hypertensionaha.120.15324.

2. Rosenthal N, Cao Z, Gundrum J, et al. Risk factors associated with in-hospital mortality in a US national sample of patients with COVID-19.JAMANetw Open. 2020;3(12): e2029058, doi:10.1001/ jamanetworkopen.2020.29058, indexed in Pubmed: 33301018.

3. Ruscica M, Macchi C, Iodice S, et al. Prognostic parameters of in-hospital mortality in COVID-19 patients-An Italian experience. Eur J Clin Invest. 2021; 51(9): e13629, doi: 10.1111/ eci.13629, indexed in Pubmed: 34184268.

4. Zheng Z, Peng F, Xu B, et al. Risk factors of critical and mortal COVID-19 cases: a systematic literature review and meta-analysis. J Infect. 2020; 81(2): e16-e25, doi: 10.1016/j.jinf.2020.04.021, indexed in Pubmed: 32335169.

5. Webb BJ, Peltan ID, Jensen P, et al. Clinical criteria for COVID-19 associated hyperinflammatory syndrome: a cohor study. Lancet Rheumatol. 2020; 2: e754-e763.

6. Shouman K, Vanichkachorn G, Cheshire WP, et al. Autonomic dysfunction following COVID-19 infection: an early experience. Clin Auton Res. 2021; 31(3): 385-394, doi: 10.1007/s10286-02100803-8, indexed in Pubmed: 33860871.

7. Goldstein DS. The extended autonomic system, dyshomeostasis, and COVID-19. Clin Auton Res. 2020; 30(4): 299-315, doi: 10.1007/s10286-020-00714-0, indexed in Pubmed: 32700055.

8. Manolis AS, Manolis AA, Manolis TA, et al. COVID-19 infection and cardiac arrhythmias. Trends Cardiovasc Med. 2020; 30(8): 451-460, doi: 10.1016/j.tcm.2020.08.002, indexed in Pubmed: 32814095.

9. Kreutz R, Dobrowolski P, Prejbisz A, et al. Lifestyle, psychological, socioeconomic and environmental factors and their impact on hypertension during the coronavirus disease 2019 pandemic. J Hypertens. 2021; 39(6): 1077-1089, doi: 10.1097/ HJH.0000000000002770, indexed in Pubmed: 33395152.

10. Grassi G, Vailati S, Bertinieri G, et al. Heart rate as marker of sympathetic activity. J Hypertens. 1998; 16(11): 1635-1639, doi: 10.1097/00004872-199816110-00010, indexed in Pubmed: 9856364.

11. Seravalle G, Grassi G. Heart rate as cardiovascular risk factor. Postgrad Med. 2020; 132(4): 358-367, doi: 10.1080/00325481. 2020.1738142, indexed in Pubmed: 32164466.

12. Liu W, Tao ZW, Wang L, et al. Analysis of factors associated with disease outcomes in hospitalized patients with 2019 novel coronavirus disease. Chin Med J (Engl). 2020; 133(9): 1032-1038, doi: 10.1097/CM9.0000000000000775, indexed in Pubmed: 32118640.

13. Paranjpe I, Russak A, Freitas JDe, et al. Clinical characteristics of hospitalized covid-19 patients in New York city. MdRxiv. 2020, doi: 10.1101/2020.04.19.20062117.

14. Zhou F, Yu T, Du R, et al. Clinical course and risk factors for mortality of adult inpatients with COVID-19 in Wuhan, China: a retrospective cohort study. Lancet. 2020; 395(10229): 10541062, doi: 10.1016/S0140-6736(20)30566-3, indexed in Pubmed: 32171076.

15. Williams B, Mancia G, Spiering W, et al. 2018 ESC/ESH Guidelines for the management of arterial hypertension. Eur Heart J. 2018; 39(33): 3021-3104, doi: 10.1093/eurheartj/ehy339, indexed in Pubmed: 30165516.

16. Grassi G, Mark A, Esler M. The sympathetic nervous system alterations in human hypertension. Circ Res. 2015; 116(6): 976-990, doi: 10.1161/CIRCRESAHA.116.303604, indexed in Pubmed: 25767284

17. Grassi G, Mancia G, Esler M. Central and peripheral sympathetic activation in heart failure. Cardiovascular Research. 2021, doi: $10.1093 / \mathrm{cvr} / \mathrm{cvab} 222$. 